Instituto Internacional de Investigación y Desarrollo Tecnológico Educativo INDTEC, C.A.

DOI: https://doi.org/10.29394/Scientific.issn.2542-2987.2018.3.8.18.334-349

OAI-PMH: http://www.indteca.com/ojs/index.php/Revista Scientific/oai

\title{
Innovación Tecnológica en la Educación Primaria
}

\author{
Autora: Luisana Sleny López Alvarado \\ Instituto Universitario de Administración y Mercadeo, CUAM \\ sleny 82@hotmail.com \\ Yaracuy, Venezuela
}

\section{Resumen}

El propósito de este ensayo es reflexionar sobre innovación tecnológica en Educación Primaria. En esta idea se utilizó el procesamiento de la información con miras a sustentar el análisis de los planteamientos teóricos relacionados con la pertinencia de la educación que se sustenta en una praxis pedagógica significativa capaz de desarrollar las capacidades e intereses, de forma que puedan apropiarse de los contenidos globales y locales en la visión de acceso a la información, considerando las oportunidades sociales. Las reestructuraciones a las que ha sido sometida la educación, se han visto influenciada por los avances en la ciencia, la tecnología y las demandas de una sociedad compleja, dinámica e incierta en los procesos de innovación educativa, que comporta la introducción de algo nuevo en el sistema educativo, modificando sus estructuras de enseñanza-aprendizaje a través de la incorporación de las Tecnologías de Información y comunicación (TIC) en el diseño curricular de la educación primaria en su intención de formar para incursión en la ciencia y tecnología a partir de uso de una amplia gama de recursos didácticos que conllevan a la innovación pedagógica. Se concluyó que la innovación tecnológica en la praxis educativa de la educación primaria, requiere además de la vocación de servicio, estudiar los nuevos paradigmas didácticos para desplegar su capacidad reflexiva y asumir el compromiso de adquirir alfabetización digital para asumir los requerimientos de una sociedad del conocimiento que es cada día más globalizada.

Palabras clave: tecnología de la información; ciencia; educación. 


\title{
Technological Innovation in Primary Education
}

\begin{abstract}
The purpose of this essay is to reflect on technological innovation in Primary Education. In this idea, information processing was used to support the analysis of the theoretical approaches related to the relevance of education that is based on a significant pedagogical practice capable of developing the capacities and interests, so that they can appropriate the global and local content in the vision of access to information, considering social opportunities. The restructuring to which education has been subjected has been influenced by advances in science, technology and the demands of a complex, dynamic and uncertain society in the processes of educational innovation, which involves the introduction of something new in education. the educational system, modifying its teaching-learning structures through the incorporation of Information and Communication Technologies (ICT) in the curricular design of primary education in its intention to train for incursion into science and technology from use of a wide range of didactic resources that lead to pedagogical innovation. It was concluded that technological innovation in the educational praxis of primary education, requires in addition to the vocation of service, to study the new didactic paradigms to display their reflective capacity and assume the commitment to acquire digital literacy to assume the requirements of a knowledge society which is increasingly globalized.
\end{abstract}

Keywords: information technology; science; education. 


\section{Introducción}

La sociedad contemporánea ha exigido una preparación que sea más especializada para aprovechar los avances de la ciencia y la tecnología. Las exigencias de una realidad, más allá de la modernidad como un derivado de la globalización. Estos cambios se inician a mitad del siglo XX, debido a una frecuente reorganización de conocimientos que demandan nuevos enfoques y maneras de abordar los avances técnicos, científicos, sociales, entre otros que responden a los requerimientos para una innovación educativa consustanciada con el acceso a la información y el conocimiento global.

Por su parte, el acceso informativo está influenciado por los avances de los conocimientos respondiendo principalmente al proceso tecnológico, social y cultural, debido al creciente auge de la comunicación entre los distintos países del mundo. Razón por la cual, en el ámbito educativo, se debe responder a las demandas de innovación de una sociedad del conocimiento capaz de adaptarse a las transformaciones requeridas en el ámbito global del siglo XXI.

La praxis escolar contemporánea, la construcción de conocimientos significativos que generen en los estudiantes un desarrollo integral. En este particular, la formación intelectual, tecnológica y social, para expresar información. Se trata de asumir una visión capaz de innovar la praxis educativa, despertando la conciencia del por qué y para qué de la acción pedagógica, lo cual representa una concepción de pertenecía a la sociedad del conocimiento.

Los aspectos señalados, permiten señalar el propósito de este ensayo es reflexionar sobre innovación tecnológica en la educación primaria. Asimismo, el procesamiento documental de la información, se realizará a través de una revisión de los aspectos más relevantes en términos de sustentar el análisis de los planteamientos teóricos relacionados con esta temática, los cuales se exponen a continuación. 


\section{Análisis de los Planteamientos Teóricos}

La calidad de la educación, puede ser concebida como un medio humano capaz de transmitir los valores socioculturales para contribuir con pertinencia educativa al desarrollo de la sociedad contemporánea. Por este motivo, la pertinencia educativa exige flexibilidad curricular capaz de desplazar el tradicional énfasis pedagógico a la transmisión de los conocimientos en los procesos de enseñanza-aprendizaje, capaz de prestar atención en los intereses, necesidades, motivaciones de los educandos en todos los niveles y modalidades del sistema educativo.

En este sentido, Tünnermann (2000): explica que "la pertinencia de la educación se juzgará en el futuro aplicando una serie de criterios, en los cuales de denotan la capacidad de las instituciones para conectarse con una diversidad de asociados en distintos niveles y trabajar con ellos en forma creativa" (pág. 183). Sugiere por lo tanto, una praxis pedagógica significativa capaz de desarrollar las capacidades e intereses, de tal forma de apropiarse de los contenidos globales, así como locales en la visión de acceso a la información, considerando las oportunidades sociales; por tanto, construye y legitima la inclusión social como la búsqueda de las soluciones posibles e impostergable para el desarrollo sustentable del país.

Por su parte, el acceso a la información ha influido en el conocimiento respondiendo principalmente al proceso tecnológico, social y cultural, debido al creciente auge de la comunicación entre los distintos países del mundo. En este sentido Samper (2002a): señala "Ios beneficios de la globalización están directamente asociados a sus posibilidades de ser realmente global, es decir, para todos $(. .$.$) y de crear un entorno para su desarrollo que respete el$ pluralismo cultural" (pág. 44). Significa un ámbito de mundialización, representa en constante transformación de la realidad educativa.

Esta contextualización, llevan a comprender que los cambios generados por los avances han propiciado la fragmentación de saberes e 
innumerables transformaciones en la concepción del conocimiento. Razón por la cual, el ámbito educativo debe responder a las demandas para adaptarse a transformaciones educativas requeridas en el ámbito del siglo XXI. Las reestructuraciones de la educación, cada vez más influenciada por los avances en la ciencia, la tecnología, de acuerdo con las demandas de una sociedad compleja, dinámica e incierta en los procesos de innovación educativa.

Los principales estudios sobre las innovaciones educativas, tienen una lógica inductiva, debido a que la mayoría analizan las experiencias que se han realizado y sobre ellas para teorizar. Estas presunciones han sido utilizadas de manera recurrente en las discusiones conceptuales y prácticas educativas algunos autores han llegado a acuñar el término como "teoría de la innovación" para referirse a sus aportes.

Del mismo modo, Tejada (1998): explica que la innovación implica "una acción que comporta la introducción de algo nuevo en el sistema educativo, modificando sus estructuras y sus operaciones de tal modo que resulten mejorados sus productos educativos" (pág. 28). Por ese motivo, se reestructuran los esquemas y las ideas fluyen en las organizaciones cuando todo se planifica, con ella se puede clarificar los diversos significados.

Precisamente, las nuevas tecnologías constituyen un eslabón de la educación actual, porque es indudable la existencia de un gran número de instituciones escolares incursionando en la adquisición de conocimientos básicos para el uso y el aprovechamiento de las herramientas computacionales. De acuerdo con Bawden (2002): este aprendizaje, se refiere a "una alfabetización electrónica" (pág. 15). Este autor, sugiere, como uno de los objetivos esenciales de la incursión tecnológica, la formación escolar de los ciudadanos para la era digital, desde una perspectiva de innovación educativa.

En correspondencia con las demandas de innovación educativa, los docentes deben reconocer el sentido de su acción, de su relación con el 
conocimiento tecno-científico, en relación con el artículo 103 de la Constitución de la República Bolivariana de Venezuela (1999), a partir de la cual se fundamentan las políticas educativas centradas en el desarrollo humano, personal, social, profesional y ético; desde una perspectiva reflexiva de la profesión docente, dando sentido a las formas de pensar, sentir y hacer de la praxis pedagógica, en términos de alcanzar la calidad educativa .

Por este motivo, el sistema educativo venezolano responde a las necesidades de innovación, con la incorporación de las Tecnologías de Información y comunicación (TIC). Así quedó expuesto en las premisas del Ministerio del Poder Popular para la Educación (2007), en el Diseño Curricular del Sistema Educativo Bolivariano como eje integrador de las políticas formativas en las que se establece:

La incorporación de las TIC's en los espacios y procesos educativos, contribuye al desarrollo de potencialidades para su uso; razón por la cual el SEB, en su intención de formar al ser social, solidario y productivo, usuario y usuaria de la ciencia y la tecnología en función del bienestar de su comunidad (pág. 58).

En este sentido, las tecnologías representan un eje integrador que impregna todos los componentes del currículo, en todos los momentos del proceso. Visto de este modo, el término innovación es ampliamente utilizado en el ámbito educativo, pero no siempre que se habla de innovar se está haciendo referencia a lo mismo. En relación con el término, se encuentra en diferentes denominaciones desplegadas en el recorrido histórico representado por Huberman (1973); Havelock y Huberman (1980); Sancho, Hernández, Carbonell, Sánchez y Simo (1993); e inclusive Blanco y Messina (2000), los cuales han utilizado esta conceptualización cuando se trata de innovaciones (a) educacionales, (b) en educación, (c) educativas o (e) con efecto educativo. En tal sentido, Rivas (1995), señala:

El significado de la innovación educativa, se manifiestan en 
todo tiempo y en todas las dimensiones de la vida. En las últimas décadas se acelera el ritmo del cambio y se multiplican (...) Cabe resaltar dos factores: el desarrollo del conocimiento científico y su difusión, la mayor participación, aspiraciones y las demandas sociales (pág. 17).

Innovar supone la construcción de nuevos aprendizajes, a su vez representan el desarrollo de procesos formativos. De esta manera, los procesos generados por la innovación, lo cual se traducen en proyectos de mejoramiento para las acciones educativas conducen progresivamente a la interrelación de la teoría y la práctica. Esta interrelación se conoce como la praxis educativa, a su vez, constituye el objeto de la innovación. Asensio (2010), sugiere como una interpretación:

En nuestros días como sinónimo de "mejorar", de "renovación positiva", de "creatividad" y, por ello, a las personas con "espíritu innovador", más que a las que simplemente realizan bien su trabajo, se las tiene por "muy valiosas", en los diferentes ámbitos profesionales. (pág. 169).

En tal sentido, los procesos de innovación tecnológica en la praxis pedagógica, implican el uso de estrategias en los métodos didácticos, mediante los cuales se trata de introducir nuevos conocimientos, concepciones y actitudes en el saber didáctico de los docentes en la sistematización pedagógica representada en gran medida, como la condición "sine qua non" de los procesos formativos, en términos de provocar los cambios en la enseñanza-aprendizaje.

Por tal motivo, De Pablos (2009): explica que es una disciplina relativamente joven que incorpora conocimientos relevantes; además de las competencias digitales, para responder a las propuestas tecnológicas desarrolladas en los centros educativos y en las prácticas educativas de los docentes en las aulas de clases conlleva una dinámica tecnológica, destinada a la innovación en la formación de los discentes. 
Por lo tanto, la tecnología educativa, conforma el ámbito didáctico donde se planean situaciones de enseñanza, implica el diseño de medios digitales, tales como el uso de la multimedia, los foros virtuales, productos audiovisuales, software asociado a procesos educativos a través del uso de estrategias didácticas como: mapas conceptuales, aprendizaje basado en casos y proyectos de aprendizajes significativos constructivistas.

En ese mismo orden de ideas, las innovaciones tecnológicas en la educación primaria, se consideran importantes para los educandos, en términos de propiciar una alfabetización digital, a partir de la cual puedan adaptarse a los cambios sociales producidos por la globalización de la información y el conocimiento, como una expresión de la reorganización de nuevos enfoques para abordar los avances técnicos, científicos, sociales, entre otros, relacionados con la concepción de sociedad del conocimiento.

En esta idea la sociedad del conocimiento, a juicio de la UNESCO (2005): describe "el desarrollo de las redes confiere al saber una importancia cada vez mayor en todas las estructuras sociales induciendo una nueva forma de dependencia tecnológica" (pág. 152). Es decir, responde al creciente auge de la comunicación entre los distintos países del mundo, lo cual representa una oportunidad para adaptarse a los requerimientos de la globalización.

En el ámbito tecnológico Samper (2002b): señala "los beneficios de la globalización están directamente asociados a sus posibilidades de ser realmente global, es decir, para todos (...) y de crear un entorno para su desarrollo que respete el pluralismo cultural" (pág. 44). En este ámbito de mundialización, la sociedad del conocimiento se ve beneficiada por el uso de la información global, reflejada en una constante transformación y restructuración.

La contextualización que antecede, lleva a comprender que existen cambios generados por los avances tecnocientíficos, los cuales han propiciado la fragmentación de saberes e innumerables transformaciones en las 
concepciones acerca de los conocimientos, competencias y actitudes para enfrentar los requerimientos contemporáneos. Razón por la cual, el ámbito educativo las transformaciones educativas, requiere de restructuraciones formativas en una sociedad compleja, dinámica e incierta.

Cebrián (2003): señala la innovación, como el medio para generar conocimientos, además de construir una nueva cultura asociada al uso e implementación de las TIC asociadas a la formación permanente. Estas ideas, por lo general aluden cambios, tanto estructurales como actitudinales; las presiones y potencialidades para la creación de nuevos significados en el proceso educativo, lo cual introducen la idea de algo diferente; aunque, este significado, deja abierta otras posibilidades en la mejora del proceso pedagógico.

La innovación educativa puede entenderse, desde una óptica amplia, como sinónimo de cambios. De León (2005): señala la creación de "procesos y estrategias, más o menos sistematizados, mediante los cuales se trata de introducir y provocar cambios en las prácticas educativas vigentes" (pág. 11). También, está asociada a la transformación que tiene un componente ideológico, cognitivo, ético y efectivo, que surgen en contextos temporales, por lo que posee una génesis propia de la evolución provocada por los avances científicos, sociales relacionada con las Tecnologías de la Información y la Comunicación (TIC).

Las TIC, son herramientas innovadoras, las cuales permiten el uso de novedosas formas de interacción entre los actores escolares en todas las etapas de la vida y de los niveles educativos. Aunado a lo anterior, la Tecnología de la Información y la Comunicación (TIC), descritas por Alvarado y Molano (2017): quienes sostienen que son "favorables a la praxis pedagógica socioeducativa, si son entendidas como estrategias didácticas complementarias" (pág. 8). Representa un conjunto heterogéneo de técnicas, programas informáticos, entre otros recursos tecnológicos que son útiles para 
la sociedad que impactan favorablemente al sector educativo.

De acuerdo con los señalamientos, el uso de las TIC como innovación educativa debe ser desarrollado por los docentes, a través del uso de herramientas instruccionales reflejada en el diseño curricular, orientado con los requerimientos técnicos para su correcta utilización para el aprendizaje de los educandos. Según Salina y Batista (2001): las nuevas tecnologías ofrecen a la educación posibilidades para el aprendizaje interactivo, a partir de esa amplia gama de recursos, se puede crear todo el ambiente necesario al aprendizaje para dar paso a la innovación pedagógica.

Otro aspecto a considerar, es la relación entre la innovación pedagógica que se articula bajo el concepto de transformación curricular se concreta en dos ámbitos de intervención: Las propuestas didácticas novedosas mejoran la praxis didáctica de los docentes, a través de la implementación de métodos constructivistas de aprendizaje colaborativo, así como el uso de estrategias tecnológicas para la elaboración de materiales complementarios que faciliten la reorganización del tiempo y espacio educativo en el empleo pedagógico de las TIC.

La pedagogía tecnológica se orienta en los planteamientos curriculares, es decir, que los maestros introduzcan modificaciones a los diversos contenidos de las materias o áreas de conocimiento del currículo formal para adecuarlos a las necesidades, intereses y contexto específico de los alumnos, ya sea a través del diseño de unidades didácticas, mapas, o de estrategias que favorezcan el logro de los aprendizajes significativos.

En lo que respecta a las innovaciones el docente utiliza en forma reflexiva y flexible conocimientos, competencias y aquellas herramientas para lograr en sus estudiantes un aprendizaje significativo, desde los planteamientos de Ausubel (2000a): hace referencia a "un contenido con estructuración lógica propia como a aquel material que potencialmente puede ser aprendido de modo significativo, es decir, con significado y sentido para el 
que lo internaliza" (pág. 120). En términos formativos, implica un proceso pedagógico integral, donde se promueva la construcción de conocimientos ajustados a las necesidades e intereses de los educandos.

Los teóricos cognoscitivistas postulan que implica una reestructuración activa de las percepciones, ideas, conceptos y esquemas en su estructura cognitiva envuelta en el proceso de enseñanza-aprendizaje pertinente con los intereses de los educandos y la realidad global contemporánea. Al respecto Ausubel (2000b), sugiere requisitos para el aprendizaje significativo, donde el estudiante debe:

Manifestar, una disposición para relacionar sustancial y no arbitrariamente el nuevo material con su estructura cognoscitiva, como que el material que aprende es potencialmente significativo para él, es decir, relacionable con su estructura de conocimiento sobre una base no arbitraria (pág. 48).

En esta idea, el desarrollo del proceso de enseñanza-aprendizaje, requiere de la elección de estrategias coherentes y efectivas que favorezcan la innovación de la praxis del docente desde la posibilidad de ofrecer mejoras en la calidad escolar. Como investigadora deduzco a la luz de los planteamientos presentados que la innovación pedagógica, siempre están presentes en la cotidianidad escolar, y no deben pasar desapercibidos, sobre todo cuando se producen como resultantes de los avances científicos, tecnológicos y sociales, provocados por los cambios epocales desde la cual se circunscribe la pertinencia de la transformación curricular en la educación primaria.

\section{Conclusiones}

A partir de una revisión documental de los constructos como eje de referencia en el desarrollo de competencias del estudiantado ante las actividades didácticas conducente a la innovación en el proceso de 
enseñanza-aprendizaje, para lo cual es necesario asumir la incorporación de las Tecnologías de Información y Comunicación (TIC) en el marco escolar, desde la comprensión de la relevancia de una educación pertinente en la sociedad del conocimiento en un mundo globalizado.

La visión de un proceso educativo globalizado, hace ineludible el compromiso de los docentes en la Educación Primaria para desarrollar en los educados la alfabetización tecnológica desde una innovación de las prácticas pedagógicas escolares en el quehacer educativo en todos los niveles y modalidades del sistema educativo venezolano.

Las innovaciones tecnológicas, por lo general aluden cambios, tanto estructurales como actitudinales. Asimismo, requiere la apropiación provechosa de las TIC en el proceso de enseñanza-aprendizaje, en todos los niveles y modalidades del sistema educativo venezolano, lo cual deja abierta la posibilidad de una mejora de la calidad de la educación primaria como la base formativa de los ciudadanos.

Los avances tecnológicos, representan al conjunto total de medios que convierten a las TIC en una innovación educativa, que ha llegado para adaptarse a los requerimientos del proceso de enseñanza-aprendizaje, debido a que la internet como portal de comunicación, información y conocimientos, ha tenido una aceptación en todos los ámbitos socioeducativos y la educación primaria no es la excepción.

Finalmente, se concluyó que la praxis de la educación primaria, requiere además de la vocación de servicio, estudiar los nuevos paradigmas didácticos para desplegar el compromiso de adquirir alfabetización digital para asumir los requerimientos de una sociedad del conocimiento que es cada día más globalizada. Se trata de promover una innovación tecnológica capaz de ir más allá de los contenidos curriculares, para despertar la conciencia de su responsabilidad de participar en las transformaciones de los modelos y estrategias educativas en la construcción de un proceso de enseñanza- 
aprendizaje pertinente y con la calidad requerida por los entornos sociales mundializados.

\section{Referencias}

Alvarado, A., \& Molano, O. (2017). Imbricaciones Tecnológicas en la Praxis

Pedagógica. Revista Scientific, 2(6), 304-321. Recuperado de:

https://doi.org/10.29394/scientific.issn.2542-2987.2017.2.6.16.304-321 Asensio, J. (2010). El Desarrollo del Tacto Pedagógico: (O la otra Formación del Educador). España: Graó de IRIF, S.L.

Ausubel, D. (2000a,b). Psicología Educativa. México: Trillas.

Blanco, R., \& Messina, G. (2000). Estado del Arte sobre las Innovaciones

Educativas en América Latina. Bogotá, Colombia: Convenio Andrés Bello.

Bawden, D. (2002). Revisión de los Conceptos de Alfabetización Informacional y Alfabetización Digital. Revista Anales de Documentación, N.ำ págs. 361-408. Recuperado de:

http://revistas.um.es/analesdoc/article/viewFile/2261/2251

De León, P. (2005). Innovación Educativa. Universidad Internacional de Andalucía. Madrid, España: AKAL, ISBN: 8446017555, págs. 164. Recuperado de: https://books.google.co.ve/books?isbn=8446017555

Cebrián, M. (2003). Enseñanza Virtual para la Innovación Universitaria. Madrid, España: Narcea.

Constitución de la República Bolivariana de Venezuela (1999). Publicada en

Gaceta Oficial N. $\mathbf{3 6 . 8 6 0}$, jueves 30 de diciembre. Caracas, Venezuela: República Bolivariana de Venezuela. Recuperado de:

http://www.inpsasel.gob.ve/moo doc/ConstitucionRBV1999-ES.pdf

De Pablos, J. (Coord.) (2009). Tecnología Educativa. La Formación del

Profesorado en la Era de Internet. Málaga, España: Ediciones Aljibe.

Recuperado de: 
http://revistas.um.es/educatio/article/viewFile/91111/87841

Havelock, R., \& Huberman, A. (1980). Innovación y Problemas de la

Educación: Teoría y Realidad en los Países en Desarrollo. Ginebra,

Suiza: UNESCO-OIE. Recuperado de:

http://unesdoc.unesco.org/images/0013/001360/136018so.pdf

Huberman, A. (1973). Cómo se Realizan los Cambios en la Educación: una

Contribución al Estudio de la Innovación. París, Francia: UNESCO-

OIE. Recuperado de:

http://unesdoc.unesco.org/images/0013/001377/137712so.pdf

Ministerio del Poder Popular para la Educación (2007). Currículo Nacional

Bolivariano. Diseño Curricular del Sistema Educativo Bolivariano.

Caracas, Venezuela: Fundación Centro Nacional para el Mejoramiento

de la Enseñanza de Ciencia, CENAMEC, ISBN: 978-980-218-281-7.

Recuperado de:

http://www.oei.es/historico/quipu/venezuela/dl 908 69.pdf

Rivas, M. (1995). Innovación Educativa. Teoría, Procesos y Estrategias.

España: Síntesis.

Salina, J., \& Batista, A. (2001). Didáctica y Tecnología Educativa para una

Universidad en un Mundo Digital, Facultad de Ciencias de la

Educación. ISBN: 9962-02-166-9. Panamá: Universidad de Panamá.

Samper, E. (2002a,b). Educación y Globalización. En Educación y

Globalización: Los Desafíos para América Latina. Volumen 1.

España: Organización de Estados Iberoamericanos para la Educación,

la Ciencia y la Cultura, OEl. [Libro en línea]. Recuperado de:

http://www.oei.es/oeivirt/temasvol1.pdf

Sancho, J., Hernández, F., Carbonell, J., Sánchez, E., \& Simo, N. (1993).

Aprendiendo de las Innovaciones en los Centros. La Perspectiva Interpretativa de Investigación Aplicada a Tres Estudios de Caso. Madrid, España: CIDE. 
Tejada, J. (1998). Los Agentes de la Innovación en los Centros Educativos. Profesores, Directivos y Asesores. ISBN: 9788487767883, págs. 232. Málaga: Aljibe.

Tünnermann, C. (2000). Pertinencia Social y Principios Básicos para Orientar el Diseño de Políticas de Educación Superior. Revista Educación Superior y Sociedad (ESS), 11(1), 181-196. ISSN: 07981228. Recuperado de:

http://ess.iesalc.unesco.org.ve/ess3/index.php/ess/article/view/364 UNESCO (2005). Hacia las Sociedades del Conocimiento. Organización de las Naciones Unidas para la Educación, la Ciencia y la Cultura. Francia: UNESCO. ISBN 92-3-304000-3, págs. 240. [Libro en línea]. Recuperado de:

http://unesdoc.unesco.org/images/0014/001419/141908s.pdf 


\section{Luisana Sleny López Alvarado \\ e-mail: sleny82@hotmail.com}

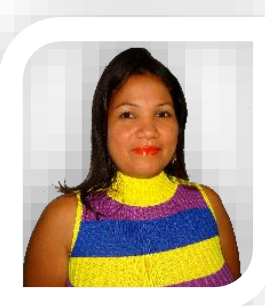

Nacida en Venezuela. Licenciada en Ciencias del

Deporte en la Universidad Experimental de Yaracuy (UNEY). Maestría en Ciencias, Mención Orientación de la Conducta en el Centro de Investigaciones Psiquiátricas Psicológica Sexología de Venezuela, Barquisimeto, estado Lara. Tesista del Doctorado en Educación, en el Convenio UPEL-CUAM, San Felipe, estado Yaracuy. Docente de aula del Ministerio del Poder Popular para la Educación 20142017. Investigadora.

El contenido de este manuscrito se difunde bajo una Licencia de Creative Commons ReconocimientoNoComercial-Compartirlgual 4.0 Internacional 